Article

\title{
Waiting for Godot: A cross sectional survey based analysis of the hydroxychloroquine prophylaxis strategy against COVID-19 in India
}

\author{
Merlin Moni, ${ }^{1}$ Thushara Madathil, ${ }^{2}$ Rahul Palabatla, ${ }^{3}$ Sabarish Balachandran, ${ }^{4}$ \\ Fabia Edathadathil, ${ }^{5}$ Georg Gutjahr, ${ }^{6}$ Sai B. Madathil, ${ }^{7}$ Rajesh Pai, ${ }^{8}$ Beena KV, ${ }^{8}$ Aveek Jayant, ${ }^{2}$ \\ Dipu T. Sathyapalan'
}

\begin{abstract}
${ }^{1}$ Department of General Medicine and Division of Infectious Diseases, Amrita Institute of Medical Sciences and Research Centre, Kochi, Kerala; ${ }^{2}$ Department of Anaesthesiology and Critical Care Medicine, Amrita Institute of Medical Sciences and Research Centre, Kochi, Kerala; ${ }^{3}$ Department of General Medicine, Amrita Institute of Medical Sciences and Research Centre, Kochi, Kerala; ${ }^{4}$ Department of Emergency Medicine, Amrita Institute of Medical Sciences and Research Centre, Kochi, Kerala; ${ }^{5}$ Department of Allied Health Sciences, Amrita Institute of Medical Sciences and Research Centre, Kochi, Kerala; ${ }^{6}$ Department of Statistics, AmritaCREATE, Amrita Vishwa Vidyapeetham, Amritapuri Kollam Kerala; ${ }^{7}$ Department of Nursing services, Amrita Institute of Medical Sciences and Research Centre, Kochi, Kerala; ${ }^{8}$ Department of Medical Administration, Amrita Institute of Medical Sciences and Research Centre, Kochi, Kerala; ${ }^{9}$ Department of General Medicine and Division of Infectious Diseases, Amrita Institute of Medical Sciences and Research Centre, Kochi, Kerala, India
\end{abstract}

\begin{abstract}
Background: India currently has the second largest burden of infections due to COVID-19. Health Care Worker (HCW) shortages are endemic to Indian healthcare. It should therefore be a huge priority to protect this precious resource as a critical component of the systemic response to this pandemic. Advisories from the Indian Council of Medical Research (ICMR) have focused on using hydroxychloroquine prophylaxis against COVID-19 in at risk HCW. This prophylaxis strategy has no evidence. In further jeopardy there appear to insubstantial attempts to build this evidence as well. In this connection, we commissioned a survey within our Institution to estimate the penetration of hydroxychloroquine (HCQ) use and use this to statistically model the impact of current ongoing studies in India. We also briefly review the literature on HCQ prophylaxis for COVID-19.

Design and methods: A structured survey designed using RedCAP application was disseminated among healthcare professionals employed at an academic referral tertiary care centre via online social media platforms. The survey was kept open for the entire month of June 2020. The survey was additionally used to statistically model the size of studies required to comprehensively address the efficacy of HCQ in this setting.

Results: 522 responses were received, of which 4 were incomplete. The ICMR strategy of 4 or more doses of HCQ was complete only in $15 \%$ of HCW in our survey. The majority of respondents were doctors $(238,46 \%)$. Amongst all category of responders,
\end{abstract}

only $12 \%(n=63)$ received the full course. A majority of those who initiated the chemoprophylaxis with HCQ turned out to be medical professionals (59/63) with neither nurse nor other categories of healthcare workers accessing the medication. The respondents of our institutional survey did not report any life-threatening side effects. Presuming efficacy as per ICMR modelling for new registry trial on the lines of the published case control study, equal allocation between cases and controls and assuming a RR of 1.3.6, the power of such a study would be very low for $n=2000$ for event rates from $2.5-12.5 \%$.

Conclusion: We report the low penetration of HCQ chemoprophylaxis among the healthcare workers of our institution. We highlight the inherent drawbacks in the study design of current national COVID related trial based on the statistical modelling of our survey results and published literature, and thereby emphasis the need of evidence-based strategies contributing to research policy at national level.

\section{Introduction \\ In December 2019, SARS-CoV-2 was identified as a cause of fatal pneumonia in Wuhan, a city in the Hubei Province of China, since then has rapidly spread and assumed pandemic proportions within a few weeks. The sheer scale of the pandemic threatens health systems. ${ }^{1,2}$ As the epidemiologic attributes of the disease are being described more accurately (the size of at risk groups and}

Significance for public health

In the context of the mounting COVID pandemic in India crippling the public health system and curtailing healthcare workers of the country, we would like to report the low hydroxychloroquine chemoprophylaxis rate from our institutional survey among healthcare workers that highlights the poor penetration of a national hydroxychloroquine prophylaxis advisory. The brief review on hydroxychloroquine (HCQ) chemoprophylaxis strategy for COVID-19 that draws on from existing literature and countrywide COVID trials reveals tenuous research designs which would be consequently informing public health policies of the nation. We emphasize on the need for continued emphasis on evidence based protective measures and rigorous efforts to build this evidence when lacking. 
asymptomatic high infectivity) the prospects are not reassuring. ${ }^{3}$

A group of French researchers first described the beneficial effect of hydroxychloroquine (HCQ) in the treatment of COVID 19. ${ }^{4}$ The paper has since been widely criticised for differential treatment of control and intervention arms and not accounting for all patients in the combination treatment arm apart from its miniscule size. Another paper from Korea has reported the use of high dose HCQ as post exposure prophylaxis for healthcare workers (HCW). This paper lacked a control arm altogether and cannot provide any inference on the repurposing of HCQ as prophylaxis against SARS CoV2 on account of its poor design. ${ }^{5}$ Likely based on this tenuous evidence the Indian Council of Medical Research (ICMR) advocated HCQ for chemoprophylaxis against SARS CoV-2 for at risk health workers in March $2020^{6}$ and has since expanded its use. ${ }^{7}$ The prevalence of this infection amongst healthcare workers in India is unclear but ranges from 1$5 \%$ in cross country survey based studies and could accelerate as the country is on course to become the most affected nation globally. ${ }^{8}$ HCQ has been the enfant terrible of this pandemic, right from the first time it was repurposed to this day. Observational data has negated benefits ${ }^{9-12}$ and has made a case for side effects (e.g., QT interval prolongation) when used for the in hospital treatment of COVID-19. ${ }^{13}$ HCQ has also been at the centre of controversies that make strident claims for revisiting the whole process of peer review for clinical research. ${ }^{14-16}$ More recently, a randomised controlled trial has since shown that HCQ when used as post exposure prophylaxis against SARS-CoV-2 is ineffective. ${ }^{17}$ Even as this literature evolves, the ICMR has since refashioned this advisory extending use to HCW working in the non Covid areas of COVID19 hospitals and to non HCW categories such as police personnel at risk of exposure. ${ }^{7}$ This advisory cites observational data obtained prospectively as also a retrospective case control dataset. The actual prospective data is not available for scrutiny. The retrospective case control study of HCQ use as chemoprophylaxis against COVID-19 has been published in the Indian Journal of Medical Research. ${ }^{18}$ This study, in our opinion, does not conclusively show that HCQ is protective against SARS-CoV-2, and therefore, cannot justify extended use as proposed. Outside India there is now a call to restrict the use of HCQ to tightly monitored environments such as clinical trials. ${ }^{19}$

Treatments and containment strategies for the pandemic, like other disease conditions, must no less be based on solidity of evidence. We point out the failure of the Indian healthcare research apparatus in seeking conclusive evidence for its targeted response to COVID-19 using the prophylactic use of HCQ against COVID19 as an example.

\section{Design and Methods}

An online structured questionnaire was developed and disseminated using the REDCAP ${ }^{\mathrm{TM}}$ platform based on a cross sectional survey study design. The questionnaire consisted of sociodemographic variables, co-morbidities, status of occupational exposure to COVID positive patients, details of hydroxychloroquine administration and side effects and willingness to participate in COVID related trials. Information on the survey was disseminated on the hospital local area network, via electronic mails to all users, by extensive use of the social medium of WhatsApp (Facebook ${ }^{\mathrm{TM}}$ ) and by word of mouth by nominated champions identified and assigned to each clinical department. We established an institutional database and present here findings on the survey of HCQ prophylaxis in our hospital.

The respondents of the survey were the healthcare staff work- ing at the hospital irrespective of their status of interaction with COVID positive patients. For dissemination, the survey proposals were circulated amongst all cadres of healthcare workers viz., physicians, surgeons, nurses, paramedical staff and non-medical workers involved in direct patient contact such as housekeeping staff, as an online link to the survey hosted on RedCAP. Each of these cadres was informed by team leaders and heads of departments including circulation by social media platforms like WhatsApp and by word of mouth, following a snow ball sampling technique. The survey was administered in English and participation was anonymous and voluntary. Non-medical staff lacking felicity in English but willing to participate were provided help in the form of transliterating questions to their mother tongue and filling the form on their behalf in their presence. The survey response data was extracted from RedCAP platform in spreadsheet format to facilitate formal analysis.

\section{Statistical analysis}

Descriptive statistics was used to summarize baseline demographics and professional characteristics. Statistical power calculations based on the data from this survey and available published literature from India were also performed. All analysis was performed on the SPSS version 17 (IBM, USA).

\section{Results}

\section{Survey results}

522 responses were received for the survey, of which 4 were incomplete. The remaining results were analysed for utilisation of prophylaxis, completeness of course of therapy, and, distribution amongst the utilised by age, comorbid conditions and category of healthcare workers. When reviewed, our limited survey depicted in Figures 1-3 highlights low penetration of HCQ prophylaxis as a sentinel finding. The ICMR strategy of deeming 4 or more doses of $\mathrm{HCQ}^{17}$ to be protective was complete only in $15 \%$ of $\mathrm{HCW}$ in our survey. The majority of respondents were doctors $(238,46 \%)$. Amongst all category of responders, a mere $12 \%(n=63)$ received the full course.

A majority of those who initiated the chemoprophylaxis with HCQ turned out to be medical professionals (59/63) with neither nurse nor other categories of healthcare workers accessing the medication. $85 \%$ of who had chosen to self-medicate per the advisory turned out to be young medical professionals, presumably healthy without comorbid conditions and resultant low risk for severe COVID disease. A fair proportion of the medicated individuals $(16 \%)$ had not however taken the loading dose. Only about $25 \%$ of those with comorbidities like systemic hypertension were on HCQ. Most respondents on HCQ prophylaxis were willing to take part in registry trials $(70 \%)$ but would not want to change over to a higher dose. Notably, our institutional data, the study from $\mathrm{ICMR}^{17}$ and $\mathrm{RCT}^{15}$ do not reveal life threatening side effects ${ }^{11}$ and, efficacy apart, there seems to be no empirical basis to consider electrocardiograms prior to and during HCQ administration, as now advised recently. ${ }^{16}$ Our analysis shows that the penetration of a national advisory on hydroxychloroquine prophylaxis was very low (Figures 1-3) based on institutional data. Even smaller proportions of those who consumed HCQ actually received what the ICMR deems protective doses viz., 4 or more doses of HCQ. ${ }^{17}$ Also, unlike the figures from Italy (HCW infection rates of $20 \%$ ) ${ }^{20}$ only $5 \%$ of the HCW in the ICMR data suffered symptomatic and confirmed SARS-CoV-2 infections. ${ }^{18,21}$ In their proposed model, 
the use of HCQ increased the risk of COVID-19 initially [adjusted odds ratio (AOR) 2.34, 95\% CI: 1.23-4.83] before a superlative protective effect afterwards for 4-5 doses (AOR 0.44, 95\% CI: $0.22-0.88$ ) and for $>6$ doses (AOR 0.04, 95\% CI: 0.01-0.16. An $\mathrm{OR}=1.5$ in the ICMR report corresponds to a relative risk (RR) of $1.36^{22}$ and an absolute risk reduction of $5.7 \%$. Per our data and their paper (Figures 1-3) the actual penetration of Covid prophylaxis now defined by a protective dose of $>4-5$ doses per their study ${ }^{17}$ is only $10-20 \%$. The actual infection rate amongst HCW is also lower at $5 \% .^{17}$

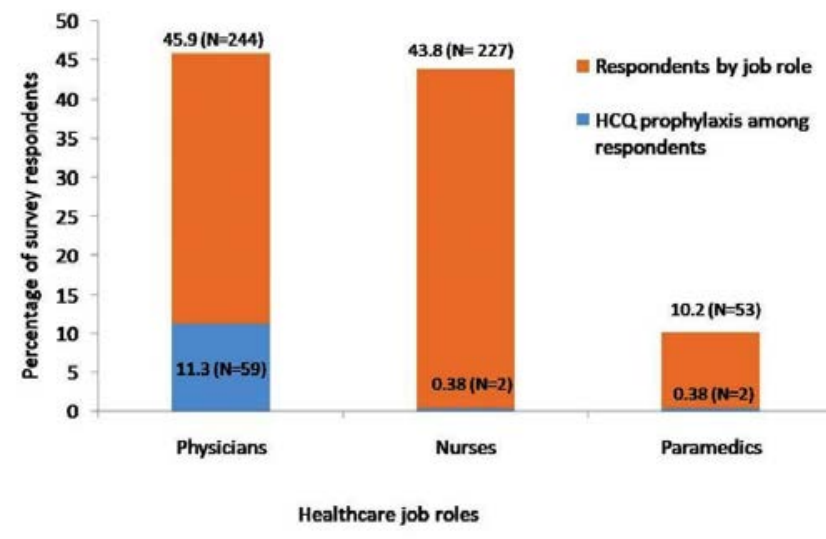

Figure 1. HCQ prophylaxis amongst healthcare workers by role : intra-institutional survey report.

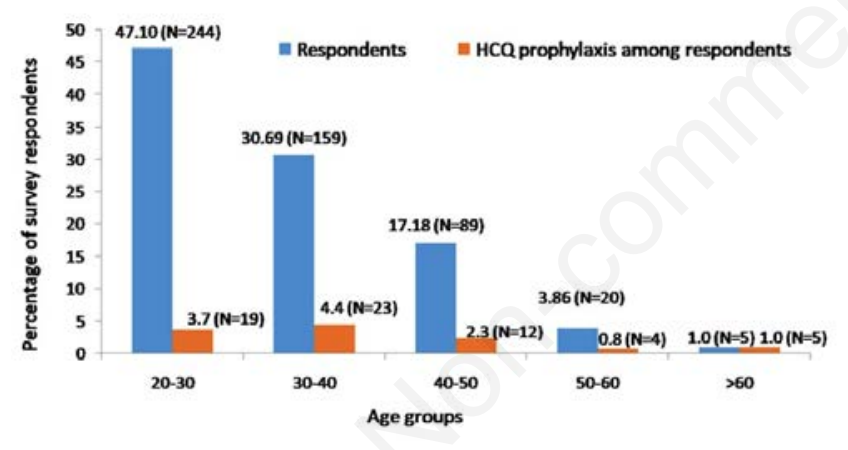

Figure 2. HCQ prophylaxis amongst healthcare workers by age: intra-institutional survey report.

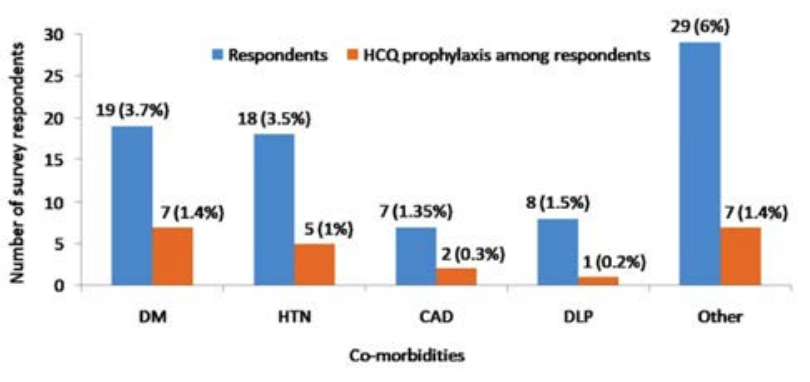

Figure 3. HCQ chemoprophylaxis distribution among patients with co-morbidities: intra-institutional survey report.

\section{Statistical modelling based on the survey}

The ICMR has now proposed a registry trial of $2000 \mathrm{HCW}$ across some centres in India (Supplementary data). We show below how this study will likely be fruitless given the low event rates overall and the low penetration of the measure.

Presuming efficacy as per ICMR modelling for new registry trial on the lines of the published case control study, ${ }^{18}$ equal allocation between cases and controls and assuming a RR of 1.3.6. Figure 4 shows that the power of such a study would be very low for $\mathrm{n}=2000$ for event rates from $2.5-12.5 \%$. This is a direct consequence of the lower HCW infection rate reported in India compared to the literature from countries like Italy. ${ }^{20}$ Now assuming more realistic allocation drawing from our survey and the ICMR paper, ${ }^{18}$ the allocation rate is closer to $12 \%$ as only about $12 \%$ of $\mathrm{HCW}$ are on a supposedly effective regimen ( $>4$ doses). Assuming this allocation for $n=2000$, the effective treatment arm is 240 . We show (Figure 5) how large the sample size in the treatment group would need to be to generate a study with a power of $90 \%$ as a function of event rate for this skewed allocation. Thus, the proposed registry trial is an exercise in futility.

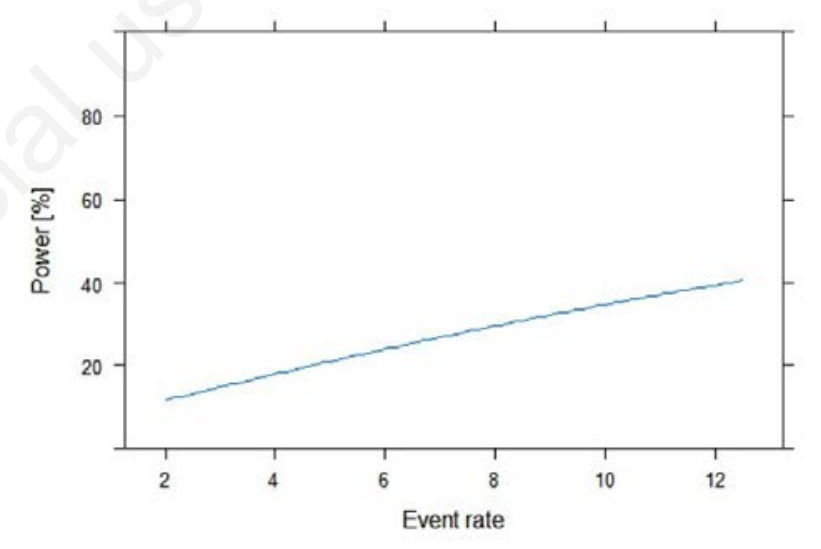

Figure 4. Sample size modelling for different event rates assuming equal allocation.

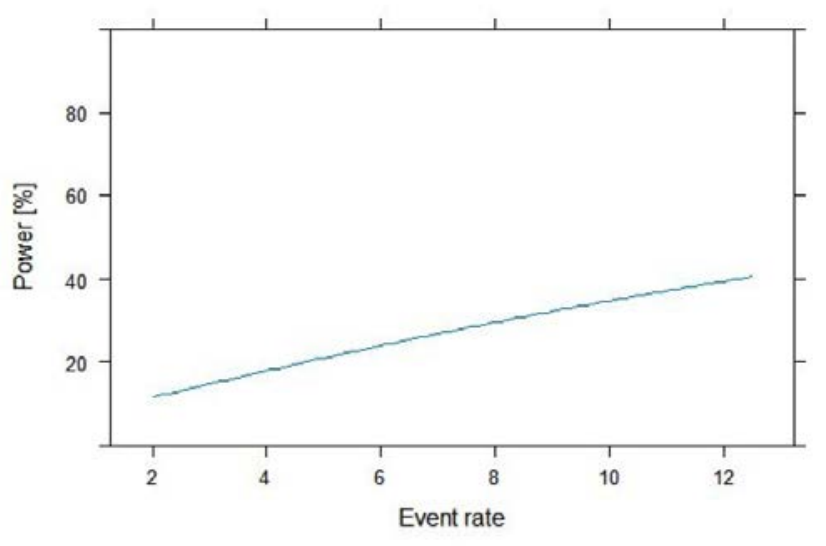

Figure 5. Sample size modelling for different event rates based on institutional 26 and ICMR reported HCQ prophylaxis penetration. 


\section{Discussion}

Healthcare workers (HCW) have considerable risks of acquiring the infection with risks described to be as high as $20 \%$ of the workforce in Italy. ${ }^{20}$ India suffers a well described chronic neglect of healthcare. With reference to the Indian workforce a recent seminal paper suggests gross understaffing, disproportionate private health representation and inadequate qualification as evident problems. ${ }^{23}$ With forecasts of high pandemic impact coupled to low healthcare resource, a prescient prophylaxis strategy makes imminent sense, when initiated early and if proven, efficacious.

Notably, India is the only country in the world to suggest a workforce wide pharmacologic prevention as early as March 2020. ${ }^{6}$ A whole two months after the lockdown nailing 130 million Indians to their homes, cases continue to surge and India is expected to face a continuum of high clinical load ${ }^{24}$ from COVID-19 with simultaneous HCW attrition ${ }^{25}$ from the pandemic itself and measures such as quarantine after workplace exposure. The state of Kerala in particular and India overall are currently in the throes of the crisis of COVID-19 with explosive increases in numbers of cases, spiralling test positivity rates and deaths even as there has been no attempt to refashion this advisory or build evidence for its support at a systemic level.

The survey has indirectly contributed to the dissemination of HCQ prophylaxis as a possible protective strategy against COVID19 amongst healthcare workers at our Institution. Despite extensive dissemination using word of mouth, emails, social media and alerts on the hospital Intranet actual responses received represented only $12-15 \%$ of total employees on the roll. This is also an indirect reminder of the grossly low penetration of the measure overall as it is plausible that those who were motivated enough to take the medication would be more easily inclined to participate in an effort to build evidence for a measure they have personally chosen. It is uncertain as to what extent our survey is representative of the larger practices in the state of Kerala or in India as a whole, limited as it is to a single centre. The electronic nature of the survey may have also limited acceptance amongst non-clinical healthcare workers such as janitorial staff and others, no less at risk from workplace exposure.

A more representative registry such as that maintained by the ICMR itself adverts to the dual difficulties of enumerating use based on registry data (only about $60 \%$ of the intended sample could be contacted for survey purposes) and about $30 \%$ of these respondents had taken the deemed efficacious dose. This strengthens our statistical modelling and hypotheses on the size of studies actually required to definitively address the efficacy or otherwise of this drug as prophylaxis. It is uncertain whether the attack rate of $\mathrm{HCW}$ and renewed motivation to attempt prophylaxis amongst $\mathrm{HCW}$ has been altered in the backdrop of rapid increase in the numbers afflicted in the period August- October 2020. In support of our contentions however other investigators from India assuming an attack rate of $10 \%$ and a modest but pragmatic and reasonable absolute risk reduction of $2.5 \%$ attest to needing a sample size of over 6000 participants to address the question of efficacy of HCQ as a prophylactic measure. ${ }^{26}$ It is however surprising that the trialists here continue to adopt the dosing scheme of the ICMR which has no evidentiary basis whatsoever and is clearly considered to deliver suboptimal doses. Simulation from the University of Minnesota ${ }^{27}$ used to drive the RCT reported in the New England Journal of Medicine from Minnesota ${ }^{17}$ and other centres in North America is the only available pharmacologic basis to provide a trial prescription for appropriate testing. If safety is a purported concern as Table 1 shows the RCT has demonstrated safety at far higher doses than the ICMR prescription with some of the participants delivered drug at distant locations and without electrocardiographic monitoring, a concern since raised about widespread unsupervised use of this molecule ${ }^{28}$. Further, the trial now proposes to commence largely in the private sector in India which has not been in the foreground of COVID-19 healthcare per scale. We surmise, based on our research experience that the proposed enrolment target will be difficult to achieve within a reasonable time given the acceleration of the pandemic making extrapolation post conclusion of limited utility. The private healthcare system also lacks organised, well-regulated research networks on the proposed scale of this trial. A single dose of HCQ has a plasma peak in 3.26 hours with a pharmacokinetic half-life of 2963 hours. Its main metabolite desethyl HCQ has a half-life of 40-50 days. ${ }^{29}$ It follows that given these kinetics it might prove difficult to enrol a rigorous placebo group given that the ICMR strategy has predated this trial by several months. The ICMR strategy could well have been adopted to a great degree in the public healthcare system which has been in the forefront of COVID-19 care in India and where the research body has huge advocacy.

Summative data from several quantitative reviews ${ }^{30,31}$ purports to the near complete lack of evidence supporting efficacy of HCQ prophylaxis overall. These reviews point to the overall lack of evidence. It is therefore logical to ask as to whether or not HCQ imparts protection beyond that offered by standard measures such as use of personal protective equipment (PPE). The overall design of the HOPE trial ${ }^{26}$ is therefore compelling as it seeks to evaluate comparative efficacy of a standard protective measure such as use of personal protective equipment (PPE) alone measured against use of PPE with HCQ as an adjunct.

In practice, however it is still contentious to define what constitutes adequate personal protective equipment and which of the constituents are more critical to protection $v s$ others. Exposures are also not standard in the face of the pandemic with aerosol generating procedures putatively of higher risk. Shortage of critical supplies such as PPE were reported in the initial stages of the pandemic but it is well known that even now certain components of the PPE arsenal such as N95 masks continue to be reprocessed: the diversity of reprocessing procedures and the lack of universal guidance from infection control bodies such as the Infectious Disease Society of America $^{32}$ also threaten to imperil standardisation of PPE as a study intervention, particularly in multicentric endeavours. The ICMR registry study has delineated some of these problems but has shied away from proposing a rigorous research design that incorporates these questions. The HOPE trial from India also leaves defining components of a PPE to institutional practice and this is likely to negatively impact study rigor. While some of these factors are difficult to control for in the situation of a pandemic standardisation in terms of defining components of a multicomponent intervention such as PPE seems a reasonable demand for trialists to seek evidence for a critical component of COVID-19 response- healthcare worker protection from this biohazard.

Table 1. HCQ dosing for prophylaxis against COVID-19: dosing schemes ICMR6,17 vs Boulware. ${ }^{15}$

\begin{tabular}{lcc} 
Day of therapy & ICMIR & Ninnesota \\
1 & $800 \mathrm{mg}$ & $1400 \mathrm{mg}$ \\
2 & $400 \mathrm{mg}$ & $600 \mathrm{mg}$ \\
\hline 3 & - & $600 \mathrm{mg}$ \\
4 & - & $600 \mathrm{mg}$ \\
\hline 5 & - & $600 \mathrm{mg}$ \\
End of week 1 & $1200 \mathrm{mg}$ & $3800 \mathrm{mg}$ \\
Week 2 onwards & $400 \mathrm{mg}$ for three weeks & - \\
Total dose of HCQ & $2400 \mathrm{mg}$ & $3800 \mathrm{mg}$
\end{tabular}




\section{Conclusion}

Our study demonstrates the poor penetration of the national advisory based HCQ prophylaxis among healthcare workers of our institution. We illustrate the poor research response of our country to the pandemic of COVID-19 using the advocacy of hydroxychloroquine prophylaxis against COVID-19 by the Indian Council of Medical Research as an example. We show how this research body (which has a stranglehold on research funding in the public health system) refuses to adapt to its own published observations, the evolving medical literature outside India and has not led Indian medical research from the front. In a resource limited system, continued loss of healthcare workers is ethically reprehensible both from the perspective of personnel lost and the health delivery apparatus as a whole. Apathy to such a critical component of COVID-19 care is emblematic of India's poor emphasis on public health and its instruments of delivery. While the private system has attempted to move beyond just care delivery to fostering clinical research its efforts can, in no substantial measure contribute to research policy at the national level given its lack of a networked collaborative research apparatus. The pandemic and this illustrative story should serve as a clarion wake up call to all those who serve and care for the health of the Indian people, in themselves, a substantial part of the human population on earth today.

Correspondence: Dipu T. Sathyapalan, Amrita Institute of Medical Sciences and Research Centre, Kochi, Kerala, India-682041.

Tel +91.484.2852250 - Fax: +91.0484.2852020

E-mail: diputsmck@gmail.com

Key words: Hydroxychloroquine chemoprophylaxis; SARS-CoV-2; COVID trial designs.

Acknowledgements: We acknowledge Information Technology services of Amrita Institute of Medical Sciences and Bri Maya for their assistance in conduct of the survey quoted in this analysis.

Funding: This work was not supported by any grants from funding agencies in the public or commercial sectors.

Conflicts of interest: Authors do not have any conflicts of interest to declare.

Ethical approval: Ethical approval for the study was sought from the Institutional Ethics Committee of Amrita Institute of Medical Sciences.

Availability of data and materials: The research data related to the study would be made available by the corresponding author on reasonable request.

Received for publication: 5 August 2020.

Accepted for publication: 12 December 2020.

oCopyright: the Author(s), 2020

Licensee PAGEPress, Italy

Journal of Public Health Research 2020;9:1888

doi:10.4081/jphr.2020.1888

This work is licensed under a Creative Commons Attribution NonCommercial 4.0 License (CC BY-NC 4.0).

\section{References}

1. Armocida B, Formenti B, Ussai S, et al. The Italian health system and the COVID-19 challenge. Lancet Public Health 2020;5:e253. doi: 10.1016/S2468-2667(20)30074-8

2. Tanne JH, Hayasaki E, Zastrow M, et al. Covid-19: how doctors and healthcare systems are tackling coronavirus worldwide. BMJ 2020;368:m1090. doi: 10.1136/bmj.m1090

3. Uddin M, Mustafa F, Rizvi TA, et al. SARS-CoV-2/COVID19: Viral Genomics, epidemiology, vaccines, and therapeutic interventions. Viruses 2020;12:526. doi: 10.3390/v12050526

4. Gautret P, Lagier J-C, Parola P, et al. Hydroxychloroquine and azithromycin as a treatment of COVID-19: results of an openlabel non-randomized clinical trial. Int J Antimicrob Agents 2020;56:105949. doi: 10.1016/j.ijantimicag.2020.105949

5. Lee SH, Son H, Peck KR. Can post-exposure prophylaxis for COVID-19 be considered as an outbreak response strategy in long-term care hospitals? Int J Antimicrob Agents 2020;55: 105988. doi: 10.1016/j.ijantimicag.2020.105988

6. Ministry of Health and Family Welfare. Advisory on the use of hydroxychloroquine in hospitalised patients with Covid-19. Accessed June 10, 2020. Available from: https://www.mohfw. gov.in/pdf/AdvisoryontheuseofHydroxychloroquinasprophyla xisforSARSC oV2infection.pdf

7. Indian Council for Medical Research. Revised advisory on the use of Hydroxychloroquine (HCQ) as prophylaxis for COVID19 infection (in supersession of previous advisory dated 23 Mar 2020). 2020. Available from: https://www.mohfw. gov.in/pdf/Revisedadvisoryontheuseofhydroxychloroquineasp rophylaxisforSARSCOVID19infection.pdf

8. Jha S, Soni A, Siddiqui S, et al. Prevalence of flu-like symptoms and COVID-19 in healthcare workers from India. J Assoc Physicians India 2020;68:27-29.

9. Geleris J, Sun Y, Platt J, et al. Observational study of hydroxychloroquine in hospitalized patients with Covid-19. N Engl J Med 2020;382:2411-8. doi: 10.1056/NEJMoa2012410

10. Tang W, Cao Z, Han M, et al. Hydroxychloroquine in patients with mainly mild to moderate coronavirus disease 2019: open label, randomised controlled trial. BMJ 2020;368:m1849. doi: 10.1136/bmj.m1849

11. Mahévas M, Tran V-T, Roumier M, et al. Clinical efficacy of hydroxychloroquine in patients with covid-19 pneumonia who require oxygen: observational comparative study using routine care data. BMJ 2020;386:m1844. doi: 10.1136/bmj.m1844

12. Borba MGS, Val FFA, Sampaio VS, et al. Effect of high vs low doses of chloroquine diphosphate as adjunctive therapy for patients hospitalized with severe acute Respiratory Syndrome Coronavirus 2 (SARS-CoV-2) infection. JAMA Netw Open 2020;3:e208857. doi: 10.1001/jamanetworkopen.2020.8857

13. Mercuro NJ, Yen CF, Shim DJ, et al. Risk of QT Interval prolongation associated with use of hydroxychloroquine with or without concomitant azithromycin among hospitalized patients testing positive for Coronavirus Disease 2019 (COVID-19). JAMA Cardiol 2020;5:1036-41. doi: 10.1001/jamacardio. 2020.1834

14. James Watson on the behalf of 201 signatories. Open letter to MR Mehra, SS Desai, F Ruschitzka, and AN Patel, authors of "Hydroxychloroquine or chloroquine with or without a macrolide for treatment of COVID19: a multinational registry analysis". Lancet. 2020 May 22:S0140-6736(20)31180-6. doi: 10.1016/S0140-6736(20)31180-6. PMID: 32450107 and to Richard Horton (editor of The Lancet). Concerns regarding the statistical analysis and data integrity. Available from: 
https://zenodo.org/record/3871094\#.X9zIs-1KhdA

15. The New York Times [Internet]. Scientists question validity of major hydroxychloroquine study. Accessed June 15, 2020. Available from: https://www.nytimes.com/2020/05/29/ health/coronavirus-hydroxychloroquine.html

16. Science Integrity Digest [Internet]. Thoughts on the Gautret et al. paper about Hydroxychloroquine and Azithromycin treatment of COVID-19 infections. Accessed May 29, 2020. Available

from: https://scienceintegritydigest.com/2020/03/24/thoughts-onthe-gautret-et-al-paper-about-hydroxychloroquine-and-azithromycin-treatment-of-covid-19-infections/

17. Boulware DR, Pullen MF, Bangdiwala AS, et al. A Randomized Trial of hydroxychloroquine as postexposure prophylaxis for Covid-19. N Engl J Med 2020;383:517-25. doi: 10.1056/NEJMoa2016638

18. Chatterjee P, Anand T, Singh K, et al. Healthcare workers and SARS-CoV-2 infection in India: A case-control investigation in the time of COVID-19. Indian J Med Res 2020. doi: 10.4103/ijmr.IJMR_2234_20

19. Qaseem A, Yost J, Etxeandia-Ikobaltzeta I, et al. Should Clinicians use chloroquine or hydroxychloroquine alone or in combination with azithromycin for the prophylaxis or treatment of COVID-19? Living practice points from the American College of Physicians (Version 1). Ann Intern Med 2020;173:137-42. doi: 10.7326/M20-1998

20. Sim MR. The COVID-19 pandemic: major risks to healthcare and other workers on the front line. Occup Environ Med 2020;77:281-2. doi:10.1136/oemed-2020-106567

21. Zheng Z, Peng F, Xu B, et al. Risk factors of critical \&amp; mortal COVID-19 cases: A systematic literature review and meta-analysis. J Infect 2020. doi: 10.1016/j.jinf.2020.04.021

22. Zhang J, Yu KF. What's the relative risk? JAMA 1998;280:1690. doi: 10.1001/jama.280.19.1690

23. Karan A, Negandhi H, Nair R, et al. Size, composition and distribution of human resource for health in India: new estimates using National Sample Survey and Registry data. BMJ Open 2019;9:e025979. doi: 10.1136/bmjopen-2018-025979

24. The Washington Post [Internet]. A frantic search for scarce hospital beds as pandemic rages in India. Available from: https:/www.washingtonpost.com/world/asia_pacific/india- coronavirus-hospital-beds/2020/06/12/7b538a8a-ab4d-11eaa43b-be9f6494a87d_story.html

25. India Today [Internet]. Help healthcare workers, Mumbai doctor appeals; warns of sharp rise in India's Covid-19 case tally. Available from: https://www.indiatoday.in/india/story/mumbai-doctor-viral-post-kem-appeal-ppe-medical-healthacre1686629-2020-06-08

26. Tirupakuzhi Vijayaraghavan BK, Jha V, Rajbhandari D, et al. Hydroxychloroquine plus personal protective equipment versus standard personal protective equipment alone for the prevention of COVID-19 infections among frontline healthcare workers: the HydrOxychloroquine Prophylaxis Evaluation (HOPE) trial: A structured summary of a study protocol for a randomized controlled trial. Trials 2020;21:754. doi: 10.1186/s13063-020-04679-3

27. Al-Kofahi M, Jacobson P, Boulware DR, et al. Finding the dose for hydroxychloroquine prophylaxis for COVID-19: The desperate search for effectiveness. Clin Pharmacol Ther 2020;108:766-9. doi: 10.1002/cpt.1874

28. Gupta A, Vijay J, Agstam S. Which QTc correction formulae (Bazett, Framingham, or Fridericia) to use for hydroxychloroquine induced QTc prolongation? Indian Pacing Electrophysiol J 2020;20:208. doi: 10.1016/j.ipej.2020.06.008

29. Food and Drug Administration. Hydroxychloroquine sulfate (Plaquenil) [package insert]. Available from: https://www.accessdata.fda.gov/drugsatfda_docs/label/2017/0 09768s037s045s047lbl.pdf

30. Cortegiani A, Ingoglia G, Ippolito $M$, et al. A systematic review on the efficacy and safety of chloroquine for the treatment of COVID-19. J Crit Care 2020;57:279-83. doi: 10.1016/j.jcrc.2020.03.005

31. Hernandez AV, Roman YM, Pasupuleti V, et al. Hydroxychloroquine or chloroquine for treatment or prophylaxis of COVID-19. Ann Intern Med 2020;173:287-96. doi: 10.7326/M20-2496

32. Infectious Diseases Society of America (IDSA). Infectious Diseases Society of America Guidelines on infection prevention in patients with suspected or known COVID-19. Available from: https://www.idsociety.org/practice-guideline/covid-19guideline-infection-prevention/ 